\title{
Neutrinoless Double-beta Decay Rates Around Mass 80 In The Nuclear Shell Model
}

\author{
K. Yanase ${ }^{* 1}$, N. Yoshinaga ${ }^{1}$, K. Higashiyama ${ }^{2}$, E. Teruya ${ }^{1}$, and D. Taguchi ${ }^{1}$ \\ ${ }^{1}$ Department of Physics, Saitama University \\ Saitama City 338-8570, Japan \\ ${ }^{2}$ Department of Physics, Chiba Institute of Technology \\ Narashino, Chiba 275-0023, Japan \\ E-mail: yanase@nuclei.th.phy.saitama-u.ac.jp \\ yoshinaga@phy.saitama-u.ac.jp koji.higashiyama@it-chiba.ac.jp \\ Teruya@nuclei.th.phy.saitama-u.ac.jp \\ Taguchi@nuclei.th.phy.saitama-u.ac.jp
}

\begin{abstract}
We carry out the nuclear shell model calculations for nuclei with $A=76$ and $A=82$, and the energy levels are compared with the experimental data for nuclei with $A=82$. We then estimate the nuclear matrix element (NME) for the $0 v \beta \beta$ decay by using the ground state wavefunctions. In order to investigate the model dependence of the NMEs, the pair-truncated shell model calculations are performed and the NMEs in terms of the wavefunctions are also calculated. It is found that the NMEs are considerably different in the models, although the number occupancies in the ground states of the parent and the daughter nuclei are almost unchanged.
\end{abstract}

The 26th International Nuclear Physics Conference

11-16 September, 2016

Adelaide, Australia

\footnotetext{
* Speaker.
} 
Table 1: Adopted single-particle energies $\varepsilon_{\tau}(\tau=v$ or $\pi)$ for neutron holes or proton particles (in MeV).

\begin{tabular}{ccccc}
\hline$j$ & $g_{9 / 2}$ & $p_{1 / 2}$ & $p_{3 / 2}$ & $f_{5 / 2}$ \\
\hline$\varepsilon_{v}$ & 0.0 & 0.5 & 1.0 & 3.0 \\
\hline$\varepsilon_{\pi}$ & 3.3 & 1.1 & 0.6 & 0.0 \\
\hline
\end{tabular}

Table 2: Adopted two-body interaction strengths. The strengths of $M P$ interaction $\left(G_{0 \tau}\right)$ are given in units of $\mathrm{MeV}$, and those of $Q P$ and $Q Q$ interactions $\left(G_{2 \tau}, \kappa_{\tau}\right.$, and $\left.\kappa_{v \pi}\right)$ are given in units of $\mathrm{MeV} / b^{4}$.

\begin{tabular}{cccccccc}
\hline & $G_{0 v}$ & $G_{2 v}$ & $\kappa_{v}$ & $G_{0 \pi}$ & $G_{2 \pi}$ & $\kappa_{\pi}$ & $\kappa_{v \pi}$ \\
\hline${ }^{82} \mathrm{Se}$ & 0.20 & 0.060 & 0.24 & 0.20 & 0.02 & 0.08 & -0.20 \\
${ }^{82} \mathrm{Kr}$ & 0.22 & 0.045 & 0.22 & 0.20 & 0.00 & 0.10 & -0.20 \\
${ }^{76} \mathrm{Ge}$ & 0.34 & 0.095 & 0.19 & 0.22 & 0.03 & 0.14 & -0.20 \\
${ }^{76} \mathrm{Se}$ & 0.32 & 0.065 & 0.07 & 0.18 & 0.03 & 0.21 & -0.20 \\
\hline
\end{tabular}

\section{SHELL MODEL CALCULATIONS FOR NUCLEAR STRUCTURE}

Systematic studies were carried out for the even-even, odd-mass, and doubly-odd nuclei in the mass region $A=80$ in terms of the shell model (SM) [1]. In the present study, four specific nuclei with $A=76$ and $A=82$ are focused for the application to the neutrinoless double beta decay. For single-particle levels, all the four $0 f_{5 / 2}, 1 p_{3 / 2}, 1 p_{1 / 2}$, and $0 g_{9 / 2}$ orbitals in the major shell between the magic numbers 28 and 50 are taken into account for neutrons as holes and protons as particles. The single-particle energies $\varepsilon_{\tau}(\tau=v$ or $\pi)$ employed in the present calculations are listed in Table 1 .

As an effective interaction, the pairing plus quadrupole $(P+Q Q)$ interaction is employed. The SM Hamiltonian is written as

$$
H=H_{v}+H_{\pi}+H_{v \pi},
$$

where $H_{v}, H_{\pi}$, and $H_{v \pi}$ represent the neutron interaction, the proton interaction and the neutronproton interaction, respectively. The interaction among like-nucleons $H_{\tau}(\tau=v$ or $\pi)$ consists of spherical single-particle energies, monopole-pairing $(M P)$ interaction, quadrupole-pairing $(Q P)$ interaction, and quadrupole-quadrupole $(Q Q)$ interaction, i.e.,

$$
H_{\tau}=\sum_{j m} \varepsilon_{j \tau} c_{j m \tau}^{\dagger} c_{j m \tau}-G_{0 \tau} P_{\tau}^{\dagger(0)} P_{\tau}^{(0)}-G_{2 \tau} P_{\tau}^{\dagger(2)} \cdot \tilde{P}_{\tau}^{(2)}-\kappa_{\tau}: Q_{\tau} \cdot Q_{\tau}:
$$

where :: denotes normal ordering. It is assumed that the interaction between neutrons and protons $H_{v \pi}$ is just given by the $Q Q$ interaction,

$$
H_{v \pi}=-\kappa_{v \pi} Q_{v} \cdot Q_{\pi}
$$

As for the single-particle basis states, the harmonic oscillator basis states with the oscillator parameter $b=\sqrt{\hbar / M \omega}$ are employed. The adopted two-body interaction strengths are listed in Table 2

Figure 1 shows the theoretical energy spectra for ${ }^{82} \mathrm{Se}$ and ${ }^{82} \mathrm{Kr}$ in comparison with the experimental data [2] 3]. The energy levels of the yrast states are well reproduced. The large energy gap between $8_{1}^{+}$and $10_{1}^{+}$state is reproduced, but the $10_{1}^{+}$and $12_{1}^{+}$states are slightly lower than the experimental energies in ${ }^{82} \mathrm{Se}$. 


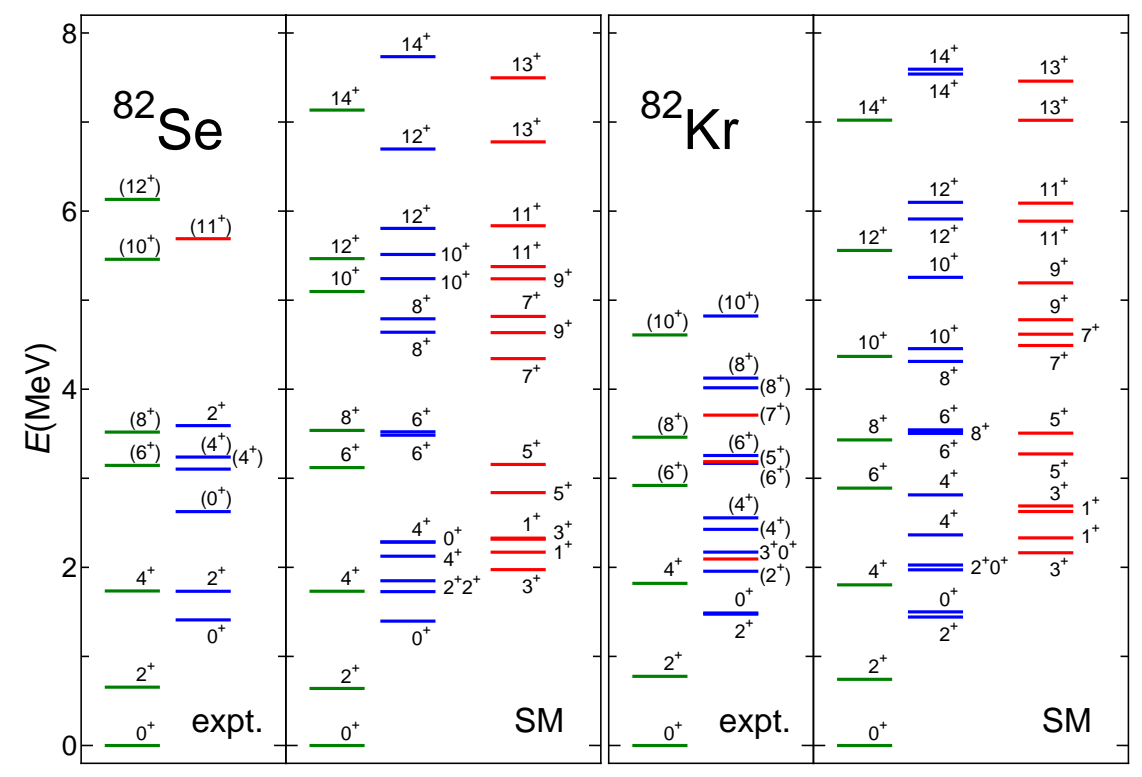

Figure 1: Comparison between the experimental energy spectra (expt.) and those of the shell model (SM) for ${ }^{82} \mathrm{Se}$ and ${ }^{82} \mathrm{Kr}$. Experimental data are taken from Ref. [2] 3].

\section{THEORETICAL FRAMEWORK AND RESULTS FOR NEUTRINOLESS DOUBLE BETA DECAY}

The inverse half-life for the $0 v \beta \beta$ decay is given as [11,12]

$$
\left[T_{1 / 2}^{(0 v)}\right]^{-1}=G^{(0 v)}\left|M^{(0 v)}\right|^{2}\left(\frac{\left\langle m_{v}\right\rangle}{m_{e}}\right)^{2}
$$

where $G^{(0 v)}$ is a phase-space factor including the axial-coupling constant $g_{A}(0)$ [13], $\left\langle m_{v}\right\rangle$ is the effective Majorana neutrino mass, $m_{e}$ is the electron mass, and $M^{(0 v)}$ is the nuclear matrix element (NME), which depends on the nuclear ground states of the parent and the daughter nuclei.

The NME for the $0 v \beta \beta$ decay is given as

$$
M^{(0 v)}=\sum_{K} M_{K}^{(0 v)}=M_{F}^{(0 v)}+M_{G T}^{(0 v)}+M_{T}^{(0 v)},
$$

where $K$ indicates either $F$ (Fermi), GT (Gamow-Teller), or $T$ (Tensor) type. Note here that we do not follow the usual definition of the Fermi-type matrix element, which is denoted in this paper as $\widetilde{M}_{F}^{(0 v)}=-\left[g_{A}(0) / g_{V}(0)\right]^{2} M_{F}^{(0 v)}$. The coupling constants $g_{V}(0)=1.00$ and $g_{A}(0)=1.25$ are adopted in this paper. The NME of each type is given by the corresponding neutrino potential $\hat{V}_{K}$ as [14

$$
M_{K}^{(0 v)}=\left\langle\Psi_{\text {fin }}\left(0_{\text {g.s. }}^{+}\right)\left|\hat{V}_{K}\right| \Psi_{\text {ini }}\left(0_{\text {g.s. }}^{+}\right)\right\rangle,
$$

where $\left|\Psi_{\text {ini }}\left(0_{\text {g.s. }}^{+}\right)\right\rangle$and $\left|\Psi_{\text {fin }}\left(0_{\text {g.s. }}^{+}\right)\right\rangle$stand for the wavefunctions of the ground states in the initial (parent) and the final (daughter) nuclei, respectively.

The $F, G T$, and $T$ type neutrino potentials can be explicitly written as 15

$$
\hat{V}_{K}=A_{s_{1} s_{2}}^{(\lambda)} \sqrt{\frac{\pi}{2 \lambda+1}} \sum_{i j} \tau_{i}^{+} \tau_{j}^{+} H_{\lambda, K}\left(r_{i j}\right)\left(Y^{(\lambda)}\left(\theta_{i j}, \varphi_{i j}\right) \cdot\left[\Sigma_{i}^{\left(s_{1}\right)} \otimes \Sigma_{j}^{\left(s_{2}\right)}\right]^{(\lambda)}\right)
$$


Table 3: The adopted parameters for each type, Fermi $(F)$, Gamow-Teller $(G T)$, and Tensor $(T)$.

\begin{tabular}{cccc}
\hline$K$ & $F$ & $G T$ & $T$ \\
\hline$\lambda$ & 0 & 0 & 2 \\
$s_{1}$ & 0 & 1 & 1 \\
$s_{2}$ & 0 & 1 & 1 \\
$A_{s_{1} s_{2}}^{(\lambda)}$ & 1 & $-\sqrt{3}$ & $\sqrt{2 / 3}$ \\
\hline
\end{tabular}

where $i, j$ represent decaying nucleons, and $\Sigma^{(s)}=1$ for $s=0$ and $\Sigma^{(s)}=\boldsymbol{\sigma}$ for $s=1$. Here, $\tau^{+}$ and $Y^{(\lambda)}$ indicate the isospin increasing operator and the spherical harmonics of rank $\lambda$ ( $\lambda=0$ for $F$ and $G T$, and $\lambda=2$ for $T$ ), respectively. The adopted coefficient $A_{s_{1} s_{2}}^{(\lambda)}$ is tabulated in Table 3 for each type $(F, G T$, or $T)$ together with the coefficients $\lambda, s_{1}$, and $s_{2}$. The orbital part of the neutrino potential, $H_{\lambda, K}\left(r_{i j}\right)$, is given in momentum representation as

$$
H_{\lambda, K}(r)=\frac{R}{g_{A}^{2}(0)} \int \frac{2}{\pi} \frac{1}{q(q+\tilde{A})} h^{K}(q) j_{\lambda}(q r) q^{2} d q,
$$

where $j_{\lambda}$ is the spherical Bessel function of rank $\lambda$. The explicit form of the neutrino potential $h^{K}$ for each type $K$ is given in Ref [14]. Here $R$ indicates the nuclear radius using the formula $R=r_{0} A^{1 / 3}\left(r_{0}=1.2 \mathrm{fm}\right)$ for mass $A$. The short-range correlations (SRC) are taken into account by multiplying the orbital potential by the Jastrow function squared, $f(r)^{2}$, with $f(r)=1-e^{-a r^{2}}(1-$ $b r^{2}$ ) adopting the Miller-Spencer parametrization: $a=1.1 \mathrm{fm}^{-2}$ and $b=0.68 \mathrm{fm}^{-2}$ [15]. In this paper the NMEs are calculated in the pure closure approximation with the closure energy $\tilde{A}=$ $\left\langle E_{N}\right\rangle-\left(\left\langle E_{I}\right\rangle+\left\langle E_{F}\right\rangle\right) / 2$, where $\left\langle E_{N}\right\rangle,\left\langle E_{I}\right\rangle$ and $\left\langle E_{F}\right\rangle$ stand for the energies of the intermediate, the initial, and the final states, respectively. Here $\tilde{A}=10.08 \mathrm{MeV}(\tilde{A}=9.41 \mathrm{MeV})$ is adopted for the decay of ${ }^{82} \mathrm{Se}\left({ }^{76} \mathrm{Ge}\right)[16$. Table 4 shows the NME in the SM calculated using the wavefunctions obtained in the previous section. The total NME in the SM is a few times smaller compared to the previous results in other models, the interacting shell models (ISMs) [17, 18], the quasi-particle random phase approximation (QRPA) [19] and the interacting boson model (IBM) [20]. There should be a caution here for the IBM results. They noted in Ref. [15] (page 6) that their results, expressed in units of $\mathrm{fm}^{-1}$, are multiplied by $2 R$, where $R=1.2 A^{1 / 3}$ is the nuclear radius in $\mathrm{fm}$. Thus their results might be reduced by half to compare them with our results. For one's reference, one half of their results are also shown in Table 4

In order to investigate the dependence of the NMEs on the wavefunctions of the ground states, the PTSM wavefunctions [6, 8] are also used to estimate the NME. We construct the $S$-pair subspace, the $S D$-pair subspace, the $S D G$-pair subspace, and the $S D G H$-pair subspace and diagonalize the Hamiltonian with the same set of parameters as used in the SM calculations. The NMEs in the PTSM are shown in Table 4 for $A=82$.

By increasing the number of types of pairs in the PTSM from $S$ to $S D, S D G$, and $S D G H$ pairs, the NMEs are gradually (not monotonically) approaching the full SM results. In fact there is a slight increase in the NME from the $S D$ to the $S D G$ cases. In the construction of the wavefunction $\left|S^{N}\right\rangle$, the $S$ pair is defined as

$$
S^{\dagger}=\sum_{j} \alpha_{j} A_{0}^{\dagger(0)}(j j)
$$


Table 4: NMEs (in dimensionless units) for the $0 v \beta \beta$ decay of ${ }^{82} \mathrm{Se}$ are given in the SM (the present study), the PTSM (the present study), the ISMs [17] 18], the QRPA [19], and the IBM [20]. For the PTSM calculations, the results within the $S D G H$-pair subspace, those within the $S D G$-pair subspace, those within the $S D$-pair subspace, and those within the $S$-pair subspace are presented. For the IBM results, one half of their results ( IBM [20]/2) are also shown for reference (see text). The axial coupling constant $g_{A}$ for each Model is given in the second column.

\begin{tabular}{ccccccc}
\hline Model & $g_{A}$ & $M_{F}^{(0 v)}$ & $\widetilde{M}_{F}^{(0 v)}$ & $M_{G T}^{(0 v)}$ & $M_{T}^{(0 v)}$ & $M^{(0 v)}$ \\
\hline SM & 1.25 & 0.281 & -0.439 & 0.648 & -0.067 & 0.861 \\
$S D G H$-pair & 1.25 & 0.358 & -0.560 & 0.707 & -0.083 & 0.982 \\
$S D G$-pair & 1.25 & 0.429 & -0.670 & 0.822 & -0.098 & 1.152 \\
SD-pair & 1.25 & 0.468 & -0.731 & 0.732 & -0.113 & 1.086 \\
S-pair & 1.25 & 0.669 & -1.045 & 1.230 & -0.139 & 1.830 \\
ISM [17] & 1.25 & & & & & 2.11 \\
ISM [18] & 1.25 & & & & & 2.18 \\
QRPA (Argonne V18) [19] & 1.27 & & -1.531 & 4.207 & -0.516 & 4.642 \\
QRPA (CD-Bonn) [19] & 1.27 & & -1.618 & 4.484 & -0.470 & 5.018 \\
IBM [20] & 1.269 & & -0.60 & 3.59 & -0.23 & 3.73 \\
IBM [20] /2 & 1.269 & & -0.30 & 1.80 & -0.12 & 1.87 \\
\hline
\end{tabular}

Table 5: NMEs (in dimensionless units) for the $0 v \beta \beta$ decay of ${ }^{76} \mathrm{Ge}$. In this case, another shell model result (ISM [21]) is also given.

\begin{tabular}{ccccccc}
\hline Model & $g_{A}$ & $M_{F}^{(0 v)}$ & $\widetilde{M}_{F}^{(0 v)}$ & $M_{G T}^{(0 v)}$ & $M_{T}^{(0 v)}$ & $M^{(0 v)}$ \\
\hline SM & 1.25 & 0.282 & -0.443 & 0.462 & -0.096 & 0.648 \\
$S D G H$-pair & 1.25 & 0.484 & -0.756 & 0.758 & -0.125 & 1.117 \\
SDG-pair & 1.25 & 0.502 & -0.784 & 0.782 & -0.129 & 1.155 \\
SD-pair & 1.25 & 0.571 & -1.893 & 1.021 & -0.134 & 1.458 \\
S-pair & 1.25 & 0.862 & -1.346 & 1.578 & -0.185 & 2.255 \\
ISM [17] & 1.25 & & & & & 2.22 \\
ISM [18] & 1.25 & & & & & 2.30 \\
ISM [21] & 1.254 & & -0.65 & 2.95 & -0.01 & 3.35 \\
QRPA (Argonne V18) [19] & 1.27 & & -1.615 & 4.715 & -0.561 & 5.157 \\
QRPA (CD-Bonn) [19] & 1.27 & & -1.713 & 5.018 & -0.510 & 5.571 \\
IBM [20] & 1.269 & & -0.68 & 4.49 & -0.23 & 4.68 \\
IBM [20] /2 & 1.269 & & -0.34 & 2.25 & -0.12 & 2.34 \\
\hline
\end{tabular}

where the structure constant $\alpha_{j}$ is determined so as to minimize the shell-model Hamiltonian,

$$
\delta\left\langle S^{N}|H| S^{N}\right\rangle=0
$$

Therefore the wavefunction $\left|S^{N}\right\rangle$ constructed by the $S$ pair corresponds to the number-conserved BCS wavefunction. In this sense the PTSM approach even only with the $S$ pairs should be superior to the mean field approaches with the pairing interaction, where only the mean value of the particle number is determined. 
The NMEs for the $0 v \beta \beta$ decay of ${ }^{76} \mathrm{Ge}$ in the SM and in the PTSM are calculated and shown in Table 5 They are compared to the other models previously cited in Table 4 Here in addition the results in a recent shell model (ISM [21]) are also shown. The total NME in the SM is a few times smaller compared to the previous results in the other models, the ISMs [17, 18, 21], the QRPA [19], and the IBM [20]. By increasing the number of types of pairs in the PTSM from $S$ to $S D, S D G$, and SDGH pairs, the NMEs are monotonically approaching the full SM results.

In order to investigate what kind of physical quantities of the wavefunctions of the ground states, we calculate the expectation value of the number operator for each orbital $j$ (the number occupancy) of the ground state. The number occupancy $v_{j}^{2}$ is defined as

$$
v_{j}^{2} \equiv\left\langle\Psi\left(0_{\text {g.s. }}^{+}\right)\left|\hat{n}_{j}\right| \Psi\left(0_{\text {g.s. }}^{+}\right)\right\rangle \text {, }
$$

where $\hat{n}_{j}$ is the number operator for the orbital $j$ and $\left|\Psi\left(0_{\mathrm{g} . s .}^{+}\right)\right\rangle$is the ground state of a specific nucleus.
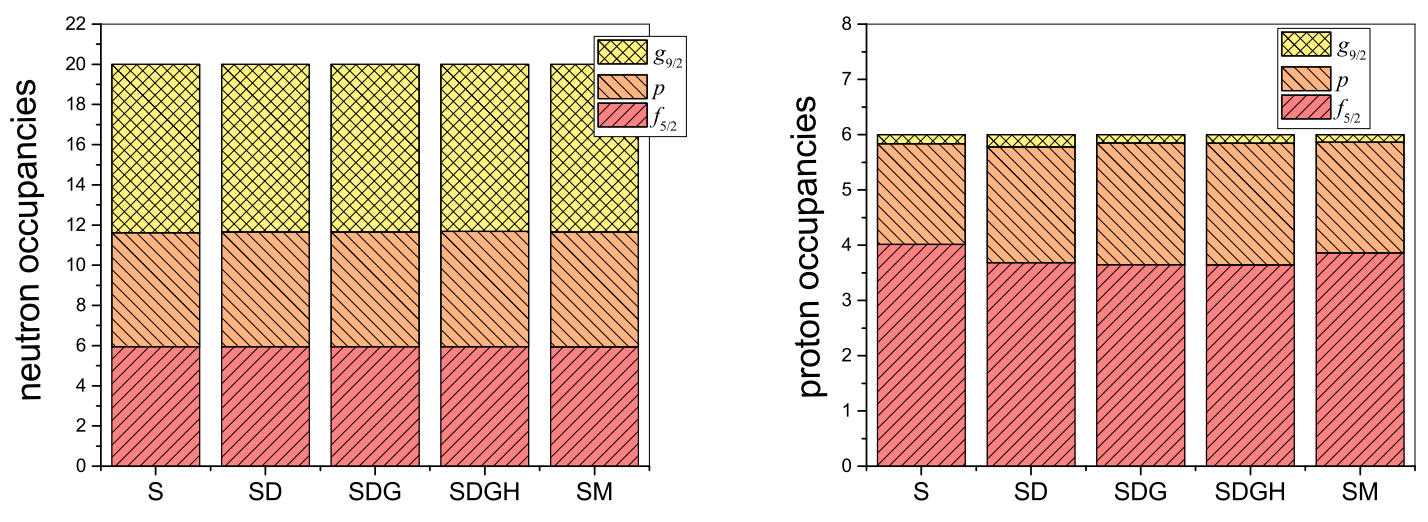

Figure 2: The occupation numbers for ${ }^{82} \mathrm{Se}$ in the SM and those in the PTSM ( $S$-pair, $S D$-pair, $S D G$-pair, and $S D G H$-pair subspaces) are shown for neutrons (left panel) and protons (right panel).
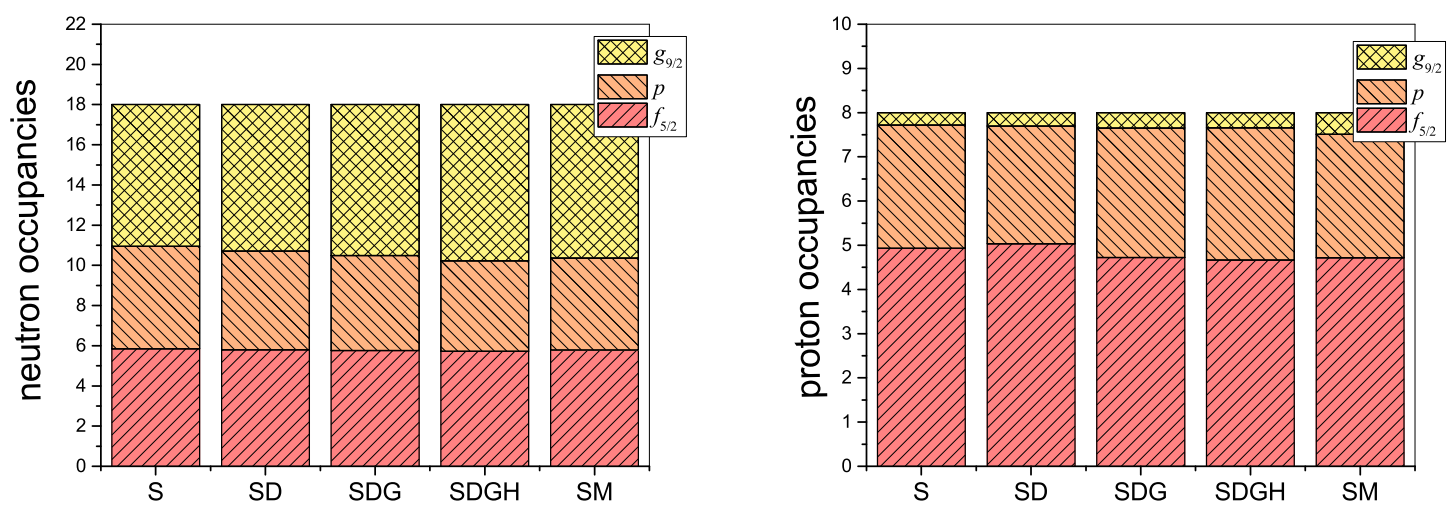

Figure 3: The occupation numbers for ${ }^{82} \mathrm{Kr}$. 
Figure 2 shows the number occupancies calculated in the SM and the PTSM for ${ }^{82} \mathrm{Se}$. The left (right) panels show the results for neutrons (protons). There are good agreements among the results in the various configuration spaces (i.e. the $S$-pair subspace, the $S D$-pair subspace, the $S D G$ pair subspace, the $S D G H$-pair subspace, and the full shell model space). In particular the neutron occupancies for ${ }^{82} \mathrm{Se}$ are little dependent on the models, because the orbitals between the magic numbers 28 and 50 are almost fully occupied in this nucleus.

Figure 3 shows the same as Figure 2 but for ${ }^{82} \mathrm{Kr}$, the daughter nucleus of the $0 v \beta \beta$ decay of

${ }^{82} \mathrm{Se}$. Good agreements among the results by the various models are also seen in this case. This fact indicates that the number occupancy does not depend sensitively on the ground state wavefunctions. On the other hand, as seen in Table 4 the NMEs change considerably according to the different models. This means that the realization of the number occupancy is not correlated to the NMEs.

\section{Summary}

In the present study, the nuclear shell model (SM) calculations are carried out for nuclei with $A=76$ and $A=82$. The energy levels of the yrast states in ${ }^{82} \mathrm{Se}$ and ${ }^{82} \mathrm{Kr}$ are shown in this paper and have good agreement with experimental data. In ${ }^{82} \mathrm{Se}$ the large energy gap between $8_{1}^{+}$and $10_{1}^{+}$ state is reproduced, but the $10_{1}^{+}$and $12_{1}^{+}$states are slightly lower than the experimental energies.

Nuclear matrix elements (NMEs) for the $0 v \beta \beta$ decay are evaluated in terms of the SM and the pair-truncated shell model (PTSM) for two cases (from ${ }^{82} \mathrm{Se}$ to ${ }^{82} \mathrm{Kr}$ and from ${ }^{76} \mathrm{Ge}$ to ${ }^{76} \mathrm{Se}$ ). Compared our results with those in other models, QRPA, IBM, and ISM, the NMEs in our SM are calculated a few times smaller, indicating the sensitivity on different wavefunctions of various nuclear models. In fact, it is shown that by expanding the model space in the PTSM from $S$-pair to $S D$-pair, $S D G$-pair, and $S D G H$-pair subspaces, the results are gradually approaching the $S M$ results and at most a few times different among the results. This means that the ground state correlations are important for the NMEs.

The number occupancy calculated for each orbital $j$ of the ground state does not depend sensitively on the ground state wavefunctions. Therefore the realization of the number occupancy is not correlated to the NMEs. In other words we do not support the validity of the NMEs calculated by using the wavefunctions, which even reproducing the experimental occupancies.

\section{Acknowledgment}

This work was supported by Grant-in-Aid for Scientific Research (C) (No. 16K05341) and (No. 25400267) from Japan Society for the Promotion of Science (JSPS), and also by Grant-inAid for JSPS Fellows Grant Number 2610429.

\section{References}

[1] N. Yoshinaga, K. Higashiyama, and P. H. Regan, Phys. Rev. C 78, 044320 (2008)

[2] G. A. Jones, et al., Phys. Rev. C 76, 054317 (2007)

[3] J. K. Tuli, Nucl. Data Sheets 98, 209 (2003)

[4] Y. Toh, Phys. Rev. C 87, 041304 (2013) 
[5] B. Singh, Nucl. Data Sheets 74, 63 (1995)

[6] K. Higashiyama and N. Yoshinaga, Phys. Rev. C 88, 034315 (2013)

[7] K. Higashiyama and N. Yoshinaga, Phys. Rev. C 83, 034321 (2011)

[8] N. Yoshinaga and K. Higashiyama, Phys. Rev. C 69, 054309 (2004)

[9] K. Higashiyama, N. Yoshinaga, and K. Tanabe, Phys. Rev. C 67, 044305 (2003)

[10] T. Takahashi, N. Yoshinaga, and K. Higashiyama, Phys. Rev. C 71, 014305 (2005)

[11] F. T. Avignone III, S. R. Elliott, and J. Engel, Rev. Mod. Phys. 80, 481 (2008)

[12] J. D. Vergados, H. Ejiri, and F. Šimkovic, Rep. Prog. Phys. 75, 10 (2012)

[13] J. Kotila and F. Iachello, Phys. Rev. C 85, 034316 (2012)

[14] F. Šimkovic, G. Pantis, J. D. Verados, and A. Faessler, Phys. Rev. C 60, 055502 (1999)

[15] J. Barea and F. Iachello, Phys. Rev. C 79, 044301 (2009)

[16] T. Tomoda, Rep. Prog. Phys. 54, 53 (1991)

[17] E. Caurier, J. Menéndez, F. Nowacki, and A. poves, Phys. Rev. Lett. 100, 052503 (2008)

[18] J. Menéndez, A. Poves, E. Caurier, and F. Nowacki, Nucl. Phys. A 818, 139 (2009)

[19] F. Šimkovic, V. Rodin, A. Faessler, and P. Vogel, Phys. Rev. C 87, 045501 (2013)

[20] J. Barea, J. Kotila, and F. Iachello, Phys. Rev. C 91, 034304 (2015)

[21] R. A. Sen'kov and M. Horoi, Phys. Rev. C 93, 044334 (2016)

[22] J. P. Schiffer, et al., Phys. Rev. Lett. 100, 112501 (2008) 